\title{
Civilisations
}

Revue internationale d'anthropologie et de sciences

humaines

39 | 1991

Japon : les enjeux du futur

\section{L'imprégnation pacifiste}

\section{Jean Esmein}

\section{OpenEdition}

Journals

Édition électronique

URL : http://journals.openedition.org/civilisations/1650

DOI : $10.4000 /$ civilisations. 1650

ISSN : 2032-0442

\section{Éditeur}

Institut de sociologie de l'Université Libre de Bruxelles

\section{Édition imprimée}

Date de publication : 30 octobre 1991

Pagination : 87-100

ISBN : 2-87263-044-9

ISSN : 0009-8140

\section{Référence électronique}

Jean Esmein, «L'imprégnation pacifiste », Civilisations [En ligne], 39 | 1991, mis en ligne le 06 juillet 2009, consulté le 19 avril 2019. URL : http://journals.openedition.org/civilisations/1650 ; DOI :

10.4000/civilisations. 1650

\section{(c) Tous droits réservés}




\section{L'IMPREGNATION PACIFISTE}

Jean ESMEIN

Ce qui paraît le plus important de dire du pacifisme japonais de nos jours ne concerne pas autant les organisations que les mentalités. L'adieu aux armes a bien paru jouer le rôle d'un thème politique, à un certain moment : pacifisme aurait pu être le nom d'un courant embrassant l'action, fabriquant une doctrine étendue à de nombreux problèmes, générant des mouvements en lutte avec les partis - absorbant même ceux-ci dans certains cas - pour monter au pouvoir. On se demanda bien sûr, après la guerre, si les grands rassemblements d'Hiroshima, visant à conjurer la politique d'armement nucléaire des puissances, n'y conduisaient pas. Ils n'allèrent pas jusque là. Ces rassemblements mobilisèrent une population importante mais disparate, puis ils cessèrent de croître. Ils restèrent conformes à un type de mouvements populaires que l'on connait bien au Japon, géants mais attachés à leur unique objet, horizontaux mais spécifiques : des mouvements dont l'existence en tout cas fait bien voir que la tendance à l'organisation verticale n'est pas la seule importante au Japon car ils peuvent même courber des politiques que les institutions voudraient faire adopter.

Il est probablement superflu de rappeler au lecteur que la vigueur des sentiments antinucléaires se renforça après l'incident dont les marins du chalutier Fukuryû Maru furent victimes en 1954, aux environs de Bikini, et que de grandes conférences internationales se tinrent ensuite au Japon chaque mois d'août à partir de l'année suivante.

Les participants et les sympathisants n'étaient autres que ceux qui, déjà en 1951, avaient apporté quatre millions et demi 
de signatures aux pétitions contre le réarmement du Japon et avaient essayé d'empêcher l'établissement d'une petite force armée de mission strictement défensive. Ils y étaient parvenus. Ils n'allaient faire sentir leur poids sur l'ensemble des politiques japonaises qu'après les troubles de 1960. Un temps s'écoule toujours avant que les "ismes" percolent. En revanche, après quelques années, le pacifisme s'installa jusque dans le sein du parti libéral-démocrate, et au gouvernement, sans même que l'on eût besoin de prononcer son nom.

Quant aux grandes conférences pour l'abolition des armes nucléraires, elles s'affaiblirent à cause de la compétition de groupes politiques désireux d'en prendre le contrôle, conflits idéologiques internes à la gauche japonaise qui considérait à la fin des années 50 que le mouvement anti-nucléaire pour la paix ne se laisserait pas identifier à d'autres qu'à elle-même.

En dépit de cela, les sentiments pacifistes des Japonais perdurèrent. Ils culminèrent pendant la guerre des Américains au Vietnam, à la fin des années 60 .

Les "ismes" ne sont pas anciens au Japon et la formule moderne de patrie intransigeante a fait à son tour une carrière brusquée, peut-être grevée de contresens. Depuis un peu avant la révolution de Meiji, l'appel aux armes, contre des étrangers ou des Japonais accusés de faire tort à des intérêts nationaux, a retenti à diverses reprises. Mais en quoi l'âme des appelés s'investissait-elle? Tandis que des initiés s'attribuaient sans partage les responsabilités de la défense, les simples soldats étaient invités à défendre l'empereur. Quand ils étaient saisis du besoin d'absolu, on leur donnait à entendre que, par leur sacrifice, ils rejoindraient le tuf végétal et les sources d'eaux vives où leurs ancêtres s'étaient déjà dissouts pour reproduire la mère nature, unique objet d'apaisement. 
Dans les carnets des soldats, petits manuels d'aphorismes pour la vie à compléter par des réflexions personnelles, on rencontrait des phrases telles que celle-ci (en fait un extrait du chapitre des Senjinkun intitulé : "Résolutions pour le temps de conscription ou de campagne") : "Lorsque le soldat part en mission, du fait même qu'il n'en revient pas toujours, il faut graver chez ses parents la pensée que l'essence d'un soldat japonais est véritablement sublime, étayant la croyance que les restes précieux qui ne peuvent leur être rendus sont les corps de fécondité des herbes, les corps de pureté des eaux". Ce naturalisme convenait peut-être à l'âme des hommes, mais pour l'adapter au nationalisme, il fallait encore quelque chose de plus. Il a des implications qui lui sont propres. On se rend compte que certaines d'entre elles auraient trouvé plus aisément leur expression dans le pacifisme que dans le nationalisme. Elles en sont là si elles existent encore à présent.

Dans une petite ville de Gumma, Nakanojô, un singulier monument aux morts a été élevé en 1961 : une pierre de deux mètres de haut sur laquelle on a gravé "stèle des sots" (Orokamono no hi). Les fondateurs sont des membres d'une association dans laquelle se retrouvent d'anciens combattants et des victimes des épurations d'après guerre.

L'interprétation de leurs volontés est complexe mais il est sûr qu'ils ont voulu attirer l'attention sur tous ceux à qui l'opinion d'après-guerre et les jugements des cours n'ont pas laissé d'autre issue que de passer pour des sots.

Cette sincérité devant les événements de la vie - y compris à l'égard de ceux qui se sont dévoués jusqu'au bout - le traitement qu'on voit ici des finalités de l'action - à mettre en pendant de celui que nous avons déjà vu pour la mort des soldats mobilisés - font penser qu'il pourrait y avoir au Japon une sorte de pacifisme natif. Paradoxalement, dans l'immédiat après- 
guerre, les autorités américaines d'occupation n'en avaient pas idée. Elles s'affairaient au recensement des crimes de guerre des Japonais. D'autre part, Ruth Benedict leur avait fourni une interprétation de la mentalité japonaise d'après laquelle le sens des rétributions reposerait sur le sens des responsabilités dans des hiérarchies vraies ou fictives, par conséquent très peu sur le poids des actions passées. Les autorités d'occupation réprouvèrent assez systématiquement l'évocation de la guerre dans l'enseignement et dans les oeuvres culturelles. Même dans les oeuvres qu'elles commandaient, le sujet était laborieusement épuré. Ainsi, elles commandèrent trois films pour servir de thème aux grandes réformes, l'un sur le libre choix politique des citoyens, un autre sur l'émancipation des femmes, et un sur la renonciation à la guerre. Le troisième échut à la firme Tôhô qui chargea Kamei Fumio de le réaliser, mais il buta sur le visa de l'état-major d'occupation et dut être remanié. Pourtant, lorsque la censure disparut, l'évocation de la guerre devint alors un thème de cinéma fréquent et les films, dans l'état où ils se présentèrent, contribuèrent beaucoup plus au pacifisme qu'à l'esprit de revanche. La contrainte idéologique des années précédentes parut avoir été vaine.

On dira pourtant qu'une masse de Japonais avait voulu la consommation de l'occupation militaire de la Mandchourie, l'extension de l'affaire de Chine contre Jiang Jieshi, l'entrée en guerre du Japon au moment des succès militaires de l'Allemagne : ils pensaient qu'un coup de poing amènerait la transformation du monde et que la conjoncture ne se reproduirait pas. Mais comme l'a dit Yamamoto Shichihei, les Japonais font toujours un choix net entre l'émeute et la fête, jamais les deux ensemble, et la plupart du temps ils optent pour la fête. Dans les élections législatives qui suivirent l'occupation de la Mandchourie, le parti Seiyûkai remporta un succès quasi total (301 élus pour 305 candidats) à la faveur d'une petite 
chanson populaire, Yonaoshi, qui célébrait "la transformation du monde" et grisa légèrement les esprits.

Oublions le passé pour revenir au pacifisme actuel et à notre impression qu'il pourrait bien être désuet.

Jusqu'à présent, les diplomates et les stratèges japonais ont pu regarder la zone stratégique dont le Japon fait partie, l'Asie Orientale, comme assez différente de l'Europe parce que les adversaires de l'Est et de l'Ouest ne se font pas face sur un front continu. Les heurts ont été intermittents, plus fréquents qu'en Europe, mais locaux (guerre de Corée, guerre du Vietnam en particulier). Sauf dans le cas de la Chine, les progrès dans la tactique d'emploi des armes nucléaires ont été inventés ailleurs. Le risque de guerre étendue a été ressenti par les populations à l'occasion de quelques crises graves, mais, généralement sans débat sur les implications que pourraient avoir des programmes d'armes nouvelles. Toutefois, au début des années 80 , les diplomates et les stratèges japonais - une partie des intellectuels à leur tour au bout de peu de temps - se sont rendu compte que la mise en ordre de bataille d'armes nucléaires par la flotte américaine dans le Pacifique nord-ouest et le resserrement des réseaux de commande de ces armes rendaient anachronique le régime de sanctuaire que les Japonais continuaient à vouloir conserver pour leur pays.

Le Ministère des Affaires Etrangères (Gaimushô), le premier, se prépara à changer de point de vue sur la politique de défense du Japon en 1980. Il observa que les alliés des Etats-Unis faisaient un effort supplémentaire, que les Soviétiques renforçaient leurs bases dans les iles Kouriles alors que ses tentatives pour contribuer à l'apaisement en Indochine étaient vaines - et que les Etats-Unis ne pouvaient faire autrement que de transférer une partie de leurs forces dans l'Océan Indien. Surtout, le Japon allait bientôt être 
regardé comme une nation riche, enrichie aux dépens des EtatsUnis, et les Américains allaient avoir de grosses dépenses pour le renouvellement de leurs armes nucléaires, proches de leur limite d'âge, alors que ces armes constituaient le parapluie nucléaire dont les alliés profitaient. Le Gaimushô admit qu'une meilleure préparation des forces armées japonaises aux missions dont les Américains avaient le souci fidéliserait ceux-ci mieux que l'indigence militaire sur laquelle on avait jusqu'alors compté pour obtenir l'entrée en ligne des Etats-Unis dès la première menace. Cependant, Reagan fut élu aux Etats-Unis et la détermination des Américains ne parut plus douteuse.

Au contraire, la marge d'initiative de l'escadre aéronavale brandissant l'arme nucléaire au voisinage des eaux japonaises et les réclamations des Américains pour une plus grande contribution des forces japonaises à sa protection devinrent trop accusées pour le pacifisme du peuple japonais.

Le premier ministre Nakasone Yasuhiro subit des pressions de l'administration américaine pour lui faire réformer la mentalité de pays protégé et le pacifisme du Japon, mais, pendant les quatre premières années de son mandat, il resta politicien. Les Américains tirèrent alors la conclusion que pendant un certain nombre d'années les Japonais n'auraient pas grand chose à leur donner pour la défense, sinon des prêts d'argent et certaines facilités techniques plus modernes pour le contrôle et les communications dans la zone du Pacifique nord-ouest. Ceci met en lumière la vrai nature des rapports américano-japonais.

Dans le nouveau régime des armes des superpuissances, que le traité de Washington entre les Etats-Unis et l'URSS a consacré, le désarmement nucléaire progressif et le parapluie nucléaire sur quoi compte le Japon vont entrer en contradiction, d'autant plus que le territoire du Japon est étroit. Le 
gouvernement japonais est favorisé parce que les armes nucléaires fixes à moyenne portée de l'Union Soviétique feront place nette comme si les réclamations japonaises avaient eu de l'effet. Les activistes du pacifisme au Japon, par contre, sont interloqués. Les armes absolues sont reléguées sur la mer et dans les airs, ce qui veut dire que la demande américaine de contribution à leur protection ne s'atténue pas. Les partisans du mouvement antinucléaire ont repris du service pour réclamer le désarmement nucléaire du Pacifique occidental. Ils ont reçu un peu de renfort. Déjà, la constitution de la République de Belau (Palao) proscrivait le séjour des armes nucléaires. Ultérieurement, la Nouvelle Zélande a pris les dispositions que l'on sait. Les pacifistes japonais se rendent compte qu'il faudrait que leur idéologie entraîne un certain nombre de pays en même temps que le leur.

Quant aux techniques de la défense nationale, on a dit quelquefois en Europe que les Japonais seraient mieux disposés envers un réarmement fondé sur les armes de l'initiative de Défense Stratégique qu'envers celui dont les Américians présentaient le modèle jusque là, parce que les armes de l'IDS seraient défensives. C'est contestable. Le progrès technique luimême entraîne un risque pour le pays hôte des armements de théâtre. De plus, comme pour les armes nucléaires, les responsables de théâtre devraient probablement se référer à la stratégie du chef d'Etat américain.

Le débat le plus sérieux se déroule donc entre les adversaires de l'arme nucléaire et les partisans d'une réforme de la défense japonaise qui s'étendrait jusqu'à l'emprunt de la force nucléaire américaine (quelques unes de leurs propres armes que les Américains déploieraient en vertu d'une procuration des Japonais, sans que les forces japonaises les stockent ou les transportent). Même des intellectuels japonais ont pris en considération cette possibilité, l'un d'eux déclarant il 
y a huit ans que le Japon ne serait pas un Etat s'il ne contrôlait pas lui-même l'emploi d'une arme nucléaire. Pourtant, le pacifisme règne et on pouvait alors voir son effet jusque dans le sein des forces armées. En avril 1972, des militaires japonais ont pétitionné leur ministre contre l'élargissement à Okinawa du stationnement des forces auxquelles ils appartiennent et il ne parait pas douteux qu'ils jugeaient que la cohabitation avec des armées équipées de l'arme nucléaire serait pire pour leur défense nationale que le maintien dans une trop étroite garnison.

De tous les obstacles qui empêchent d'établir la défense du Japon à un niveau peu en rapport avec la force du pays, le plus important vient sans doute de l'esprit du peuple japonais. Dans leur majorité, les Japonais continuent à penser sans doute qu'ils en ont fini avec la guerre. La leçon américaine a suffi. Que les Américains montent la garde et renforcent leurs armes autour du Japon s'ils pensent que la situation stratégique l'exige! Le Japon peut même leur donner de l'argent pour cela s'ils en demandent. L'expérience d'un gouvernement qui a été subverti par l'armée, laquelle a mené le peuple dans la pire guerre, est encore aujourd'hui stérilisante.

En fin de compte, l'imprégnation du pacifisme a pénétré jusqu'à la conscience sociale. Elle a fini par transformer l'éthique au point que certaines valeurs s'en trouvent contaminées. En particulier, les hommes qui servent la nation dans l'armée mériteraient un traitement plus juste que celui qui leur est fait. Le lecteur en jugera par l'histoire qui va suivre. Elle met en lumière les conséquences des préjugés du public contre l'armée et ces préjugés donnent la mesure de l'imprégnation pacifiste.

En juillet 1988, un abordage eut lieu en baie de Tôkyô, dans le chenal d'Uraga, entre un sous-marin en surface et un navire 
de promenade, chalutier hâtivement transformé pour offrir à des citadins des excursions en mer. L'accident fit trente morts. Il apparaît que, dès le début et pendant un intervalle de temps très long, les médias ont inspiré l'idée que les marins de l'Etat étaient coupables - et même indifférents au sauvetage des autres - mais aussi que l'officier qui commandait le sous-marin a été mis en quarantaine, privé de parole, de contacts avec sa famille, comme si le gouvernement avait tenu à ce que l'opinion ait d'abord son content, puisque de toute façon, c'était une sale affaire.

Le treize juillet 1988 vers 15 h 30 , une petite force navale japonaise rentrait à Yokosuka après une période d'exercices. Le sous-marin Nadashio avait reçu liberté de manoeuvre. Un petit navire de plaisance, Daiichi Fujimaru, chalutier transformé en bateau d'excursions, chargé de touristes et de pêcheurs, se dirigeait vers la sortie de la baie de Tôkyô. Les deux étaient distants de deux mille cinq cent mètres lorsque leurs deux commandants s'aperçurent que leurs routes se croisaient. Le sous-marin allait au port militaire; il gagnait vers l'ouest. Le chalutier était dans le chenal d'Uraga, en marche vers le sud, près de la bordure ouest du chenal, mais les eaux sont assez profondes devant le port et son faible tirant d'eau lui permettait de se rapprocher de terre, au besoin.

Le sous-marin ralentit sa marche pour un voilier, puis il rétablit sa vitesse à onze noeuds. La distance se réduisit à sept cents mètres. Le sous-marin se rendit compte qu'il faisait route de collision. Comme il le voyait par tribord avant, que le chalutier de son côté le voyait par bâbord avant, c'était au commandant du sous-marin de manoeuvrer. Il fit mettre la barre à droite en grand, signala sa manoeuvre par un coup de sifflet, ensuite stoppa les moteurs et il commanda : "arrière toute". A six cents mètres de lui, le chalutier vint à gauche, c'està-dire vers le sous-marin; probablement arrêta-t-il aussi sa 
machine, mais sa décélération fut lente. L'avant du chalutier monta sur le ballast arrondi du sous-marin, à tribord. Il avait tendance à déjauger car la cale à poisson avait été transformée en salon sans égard au bilan longitudinal des poids. Le chalutier gîta très fort, embarqua beaucoup d'eau et chavira. Il coula vite parce que les panneaux étanches n'étaient pas fermés...

La manoeuvre du chalutier déçoit les gens de mer. Tels que les faits sont présentés, il aurait été bien plus convenable de virer à droite pour ne pas réduire trop vite la distance avec le sous-marin, d'autant plus que celui-ci avait sifflé un coup, prévenant de son abattée sur tribord.

Un certain temps après l'accident, des médias envoyèrent leurs hélicoptères qui filmèrent des marins se croisant les bras sur le pont. A ce moment là, le repêchage des survivants du chalutier était terminé. Néanmoins, les nouvelles du soir et du lendemain firent entendre que les marins de guerre du Japon pourraient bien être enclins à la même arrogance que les militaires d'autrefois. Ensuite, dès que les survivants purent sortir de l'hôpital, ils furent harcelés par des journalistes anticipant à leur manière les questions du tribunal maritime qui est seul vraiment compétent. La tournure de ces interviews entraîna l'opinion à penser que le sous-marin était coupable de non-assistance à des personnes en danger.

Du 24 juillet au 15 septembre, les médias eurent toute latitude pour gloser la faute des marins de guerre car on ne donna pas la parole à ceux-ci. Même rentré à sa base, le commandant demeura confiné dans son sous-marin pendant trois semaines - jusqu'à la reconstitution de l'accident le 13 août - et il y a lieu de se demander si les droits de l'homme n'ont pas subi en cela un accroc. Quand enfin il s'exprima à son tour devant un journaliste, il ne dit rien de la responsabilité du Daiichi Fujimaru. Il dit qu'il s'était soumis à l'interview en 
considérant que le jugement qui serait rendu devait être exact et complet : "Du fond du coeur je prie pour que les personnes qui sont mortes aient une bonne vie dans l'au-delà. En tant qu'acteur de cet incident, je suis pénétré de responsabilité", conclut-il.

On a l'impression que pour des faits de cette sorte au Japon, le drame ne pouvait être enterré qu'au prix de la pénitence des marins de guerre, quoi que dît le droit en leur faveur. Cependant, l'affaire a accru la défiance du public à l'égard des forces d'autodéfense étant donné la façon dont on l'a laissée se développer.

Si le gouvernement, le parti libéral-démocrate et les politiciens trouvent bon que les choses se passent ainsi, sans que les marins se défendent avant l'audience du tribunal maritime, c'est certainement parce que le pacifisme est encore une valeur qui pèse lourd dans les bulletins de vote du peuple, nécessaires pour gouverner. 


\section{BIBLIOGRAPHIE}

COSTELlO, William. Democracy vs. Feudalism in Postwar Japan. Tôkyô, Itagaki Shoten, 1948.

HAGA, Tôru. Hôhô to shite no Minshû in Nihon Dolensho Shimbun $\mathrm{n}^{\circ} 1750$, mars 1974.

JOECK, Noël. Strategic Consequences of Nuclear Proliferation in South Asia. London, Frank Cass and Co., 1986.

KUGAI, Saburô. The Nuclear Umbrella in East Asia in Exterminism and Cold War, London, Verso, 1982.

MAGAMI, Hiroshi. Doko mo kakanakatta Shôtotsu Gemba 15 Funkan $\mathrm{n}^{\circ}$ Shinjitsu in Bungeishunjû , 1988, $\mathrm{n}^{\circ} 10$.

NAKAMURA, Tesu, MARUYAMA Masao, TSUJI, Kigoaki, SEIJIGAKU Jiten, Tôkyô, Heibonska, 1954.

OOKA Shôhei, YUKI, Shôji, Heishi to Kokka in Chûôkôron 1972, n 4.

TAKAYANAGI, Sakio. La perception japonaise des relations extérieures : le dilemme et les choix in Pouvoirs , 1985, $n^{\circ} 35$.

TSURUMI, Shunsuke. A Cultural History of Postwar Japan, London, KPI Ltd., 1987.

YAMASHITA, Keisuke, "Nadashio O. Kanchô" Zenkokuhaku in Bungeishunjû , 1988, $n^{\circ} 10$. 


\section{SUMMARY}

Pacifism in Japan is very old, with roots going back a number of centuries. After the demise of militarism, the immediate post-war period saw it rise phoenix-like from the ashes. For the last 30 years, the pacifist movement has kept alive the opposition to the rebirth of militarism and has opposed the liberal party's policy of collaboration with the US. In fact, Japan has agreed to an increasingly tighter integration into the US defence strategy in the Pacific, even to the point of accepting the storage of nuclear weapons on her territory. Furthermore, as time has passed and Japan's power as an economic giant has increased, a certain number of Japanese intellectuals have developed the idea that a truly sovereign state must be armed. And this is mirrored in the ever-widening public acceptance of the build-up of the Self-Defence Forces. Nevertheless, a recent accident involving a nuclear submarine and a trawler demonstrated how delicate the whole matter remains, and that pacifism remains a sentiment firmly rooted in the Japanese psyche. 